\title{
Predictive score for the development or progression of Graves' orbitopathy in patients with newly diagnosed Graves' hyperthyroidism
}

\author{
Wilmar Wiersinga 1, *, Miloš Žarković2, *, Luigi Bartalena ${ }^{3}$, Simone Donati $^{4}$, Petros Perros $^{5}$, \\ Onyebuchi Okosieme ${ }^{6}$, Daniel Morris ${ }^{7}$, Nicole Fichter $^{8}$, Jurg Lareida ${ }^{8}$, Georg von Arx $^{8}$, Chantal Daumerie ${ }^{9}$, \\ Maria-Christina Burlacu ${ }^{9}$, George Kahaly ${ }^{10}$, Susanne Pitz ${ }^{11}$, Biljana Beleslin², Jasmina Ćirić ${ }^{2}$, Goksun Ayvaz ${ }^{12}$, \\ Onur Konuk ${ }^{13}$, Füsun Baloș Törüner ${ }^{12}$, Mario Salvi ${ }^{14}$, Danila Covelli ${ }^{14}$, Nicola Curro ${ }^{15}$, Laszlo Hegedüs ${ }^{16}$ and \\ Thomas Brix ${ }^{16}$ on behalf of EUGOGO (European Group on Graves' Orbitopathy)
}

${ }^{1}$ Department of Endocrinology and Metabolism, Academic Medical Center, University of Amsterdam, Amsterdam, Netherlands, ${ }^{2}$ Department of Endocrinology, School of Medicine, University of Belgrade, Belgrade, Serbia, ${ }^{3}$ Endocrine Unit, Ospedale di Circolo, ${ }^{4}$ Department of Medical and Surgical Sciences, School of Medicine, University of Insubria, Varese, Italy, ${ }^{5}$ Department of Endocrinology, Royal Victoria Infirmary, Newcastle upon Tyne, UK, ${ }^{6}$ Department of Endocrinology, Institute of Molecular and Experimental Medicine, Cardiff University School of Medicine, Cardiff, UK, ${ }^{7}$ Cardiff Eye Unit, University Hospital of Wales, Cardiff, UK, ${ }^{8}$ Department of Ophthalmology, Interdisciplinary Centre for Graves' Orbitopathy, Olten, Switzerland, ${ }^{2}$ Department of Endocrinology, Université Catholique de Louvain, Cliniques Universitaires Saint-Luc, Brussels, Belgium, ${ }^{10}$ Department of Medicine I, Johannes Gutenberg University Medical Center, Mainz, Germany, ${ }^{11}$ Orbital Center, Ophthalmic Clinic, Buergerhospital, Frankfurt, Germany, ${ }^{12}$ Departments of Endocrinology and Metabolism, ${ }^{13}$ Ophthalmology, Faculty of Medicine, Gazi University, Ankara, Turkey, ${ }^{14}$ Graves' Orbitopathy Unit, Department of Clinical Science and Community Health, Fondazione Ca'Granda IRCCS, University of Milan, Milan, Italy, ${ }^{15}$ Department of Ophthalmology, Fondazione IRCCS Ca'Granda Ospedale Maggiore Policlinico, Milan, Italy, and ${ }^{16}$ Department of Endocrinology and Metabolism, Odense University Hospital, University of Southern Denmark, Odense, Denmark

Correspondence should be addressed to M Žarković

Email

milos.zarkovic@med.bg.ac.rs

\begin{abstract}
Objective: To construct a predictive score for the development or progression of Graves' orbitopathy (GO) in Graves' hyperthyroidism $(\mathrm{GH})$.

Design: Prospective observational study in patients with newly diagnosed GH, treated with antithyroid drugs (ATD) for 18 months at ten participating centers from EUGOGO in 8 European countries.

Methods: 348 patients were included with untreated GH but without obvious GO. Mixed effects logistic regression was used to determine the best predictors. A predictive score (called PREDIGO) was constructed.

Results: GO occurred in 15\% (mild in $13 \%$ and moderate to severe in 2\%), predominantly at 6-12 months after start of ATD. Independent baseline determinants for the development of GO were clinical activity score (assigned 5 points if score $>0$ ), TSH-binding inhibitory immunoglobulins (2 points if TBII 2-10 U/L, 5 points if TBII > 10 U/L), duration of hyperthyroid symptoms ( 1 point if $1-4$ months, 3 points if $>4$ months) and smoking ( 2 points if current smoker). Based on the odds ratio of each of these four determinants, a quantitative predictive score (called PREDIGO) was constructed ranging from 0 to 15 with higher scores denoting higher risk; positive and negative predictive values were 0.28 (95\% Cl $0.20-0.37)$ and 0.91 (95\% Cl 0.87-0.94) respectively. Conclusions: In patients without GO at diagnosis, $15 \%$ will develop GO (13\% mild, $2 \%$ moderate to severe) during subsequent treatment with ATD for 18 months. A predictive score called PREDIGO composed of four baseline determinants was better in predicting those patients who will not develop obvious $\mathrm{GO}$ than who will.
\end{abstract}

European Journal of

Endocrinology

(2018) 178, 635-643
Published by Bioscientifica Ltd. 


\section{Introduction}

A subset of patients with Graves' hyperthyroidism (GH) also have Graves' orbitopathy (GO) (1). Development of $\mathrm{GO}$ is feared by patients because it substantially impairs quality of life due to interference with visual functions and unmistakable changes in appearance (2). Fortunately, not all patients with Graves' disease develop GO. At the time of diagnosing $\mathrm{GH}$, about $75 \%$ have no GO (3). However, GO may become evident after starting treatment for hyperthyroidism. In patients with newly diagnosed GH presenting without obvious GO, it might be useful if one could estimate the risk of developing GO. In patients with sufficiently high risk, one may prefer treatment with antithyroid drugs or total thyroidectomy rather than radioactive iodine in view of the adverse effect of 131I on the course of GO $(4,5,6)$. In high-risk patients, one could also envisage prophylactic treatment with selenium, which does prevent worsening of GO in mild cases (7). Therefore, a prospective study was initiated in patients with newly diagnosed GH without overt GO, aiming at constructing a predictive score for developing GO during antithyroid drug treatment. Well-known risk factors for GO are smoking (8) and high serum concentrations of TSH receptor antibodies $(9,10)$. Age, gender and thyroid function were also evaluated as putative predictors, because older age, male sex and biochemically severe hyperthyroidism have all been associated with more severe $\mathrm{GO}(5,11,12)$.

Patients with overt GO were excluded, but mild eyelid swelling and mild conjunctival redness were no reason to exclude patients. The rationale behind this is that minimal swelling and redness of the eyes in the absence of other ophthalmological signs compatible with GO are not necessarily diagnostic of GO: some swelling and redness of the eyes are common in many subjects regardless of Graves' disease. Thus, minimal swelling and redness in our study participants at baseline was considered as either a finding unrelated to the diagnosis of Graves' disease, or representing very mild, almost subclinical GO. However, the presence of even minimal swelling and redness score 2 points on the clinical activity score (CAS), a widely used tool to assess activity of GO (13). That score by itself should not be taken as evidence for the existence of GO as it should be realized that the CAS is not specific for the diagnosis of GO. Any inflammatory eye or orbital disease may result in swollen, red or painful eyes, and thus, in positive CAS scores. Therefore, it was decided to include the evaluation of baseline CAS as a putative predictor of developing GO. Furthermore, the predictive value of the Vancouver Orbitopathy Rule was assessed, a short questionnaire for self-reporting of GO symptoms in patients with a recent diagnosis of GH (14).

\section{Subjects and methods}

\section{Patient recruitment}

Patients were recruited from ten participating centers of the European Group on Graves' Orbitopathy (EUGOGO) in the period May 2009-May 2014. Inclusion criteria were untreated GH, absence of overt GO and planned treatment with antithyroid drugs for 18 months. Definition of GH was (a) decreased TSH, elevated FT4 and/or FT3 and (b) diffusely enlarged thyroid gland (either by palpation or ultrasonography) and/or homogeneous thyroid uptake at scintigraphy. Antithyroid drugs could be administered according to either the titration method or the block-andreplace regimen, in line with standard policies of each participating center.

The mere presence of mild conjunctival redness and/or mild eyelid swelling (according to Figs 6 and 7 respectively in the color atlas by Dickinson and Perros) (15) was not a reason to exclude patients, provided other signs suggesting GO were absent. Exclusion criteria were (1) previous or planned treatment with 131I or thyroidectomy, (2) presence of GO, defined as one or more of the following eye changes: (a) soft tissue changes (moderate or severe eyelid/conjunctival redness, moderate or severe eyelid/periorbital swelling) as depicted in the color atlas (15); (b) proptosis above the upper normal limit (Asians $18 \mathrm{~mm}$, Caucasians $20 \mathrm{~mm}$, blacks $22 \mathrm{~mm}$ ); (c) diplopia (intermittent, inconstant or constant); (d) decreased visual acuity attributable to GO, (3) drugs interfering with natural course of GO (e.g. steroids, cytokines, anticytokines, thiazolidinediones, selenium), (4) drugs interfering with thyroid function (e.g. amiodarone, lithium, iodine supplements), (5) drug or alcohol abuse, (6) no informed consent.

Approval of institutional review boards or local ethical committees was not deemed necessary because the study protocol did not require additional procedures beyond those done in the delivery of usual care. Nevertheless, consent has been obtained from each patient after full explanation of the purpose and nature of all procedures. 


\section{Putative determinants}

Putative determinants for developing GO were assessed at baseline before starting antithyroid drugs. They included age and gender, family history of autoimmune thyroid disease, other autoimmune diseases in the patient, duration of hyperthyroid symptoms until start of antithyroid drugs, biochemical severity of hyperthyroidism (TSH, FT4, FT3), immunological severity (TBII, TPOAb), smoking behavior (never smoker, ex-smoker, current smoker), CAS and the Vancouver Orbitopathy Rule (14). The Vancouver Orbitopathy Rule consists of five questions: (1) do you have redness in your eyes or eyelids? (2) do you have swelling or feeling of fullness in one or both of your upper eyelids, (3) do you have bags under the eyes? (4) do your eyes seem to be too wide open? (5) is your vision blurry, even with glasses/contacts? If answers to questions 2 or 3 was 'yes' and in addition if any of the answers 1, 4 or 5 was 'yes', the result is labeled positive (sensitivity 0.76 and specificity 0.82 for the diagnosis of GO in patients with GH) (14).

\section{Study protocol}

In this prospective cohort study, baseline assessment of eye changes and putative determinants were performed mostly by endocrinologists. Soft tissue changes were recorded according to the color atlas (15), lid aperture in $\mathrm{mm}$ in midline by ruler, proptosis in $\mathrm{mm}$ by Hertel exophthalmometer; CAS scores were at a scale from 0 to 7 (13). Patients were asked about double vision (none, intermittent, inconstant, or constant), decreased visual acuity and disturbed color vision (if present, patient was referred to the ophthalmologist). Antithyroid drugs were started after blood sampling.

Follow-up visits at 6-12-18 months included blood sampling and re-assessment of smoking behavior and eye changes, again by the endocrinologist.

End-points of study were development of GO as defined under exclusion criterion 2 (GO was also diagnosed if proptosis values had increased by more than $2 \mathrm{~mm}$ ) or otherwise at 18 months when antithyroid drugs were discontinued. Premature stops could happen if 131I therapy or thyroidectomy was performed earlier than 18 months or in case of severe intercurrent illness. Discontinuation of antithyroid drugs before 18 months or pregnancy $(n=2)$ were no reason to exclude patients.

\section{Statistical analysis}

Assays of thyroid function and thyroid antibodies were done at each center in the local laboratory. As different assays with different reference values were used, all obtained results were standardized by dividing obtained results by the upper normal limit of that assay, and then multiplied by the most common upper limit of the reference range. Normally distributed data are reported as mean \pm s.D., while data not normally distributed are reported as median with range. Categorical variables are recorded as numbers and percentage. TBII was measured by second generation assays.

The objective of the statistical analysis was to identify determinants of developing GO and to construct a predictive score (called PREDIGO). Comparisons were made between patients who did or did not develop GO during the 18-month follow-up period. Odds ratios for developing GO were calculated for each of the putative determinants assessed at baseline, using univariate logistic regression. For multivariate analysis, mixed effects logistic regression was performed to find which factors were independently related to GO. R and Ime4 software were used $(16,17)$. As fixed effects, clinical, laboratory and demographic data were entered, and as a random effect intercept for center. Because of the wide distribution of the data, logarithmic transformations for TSH, FT4, TPOAb, TBII and duration of hyperthyroid symptoms were used. To find the optimal model, multiple models were created and compared. $P$ values were obtained by likelihood ratio tests of the model with the effect in question against the model without the effect in question. 95\% confidence intervals for effects were calculated using the Wald method. For the predictive score, significant parameters were used as obtained by the logistic regression. Number of score points assigned to each parameter was calculated using the Framingham method (18). The sum of the score points constituted the PREDIGO score. Optimal cut-off for the PREDIGO score was obtained by analysis of the ROC curve and minimizing distance between ROC plot and point $(0,1)(19)$. The characteristic of the predictive score was assessed by bootstrapping.

\section{Results}

392 patients were recruited. Eleven patients were subsequently excluded (9 opted for thyroidectomy and 1 for radioactive iodine, and 1 patient suffered a stroke). Another 33 patients were lost to follow-up, leaving 348 patients for analysis. Characteristics of the study population are listed in Table 1 . Two patients became pregnant during the study period; one of them 
Table 1 Baseline characteristics of 348 patients with Graves' hyperthyroidism treated with antithyroid drugs for 18 months: 295 patients remained without GO and 53 patients developed GO during 18 months of follow-up.

\begin{tabular}{|c|c|c|c|c|}
\hline & 348 patients & $\begin{array}{l}295 \text { patients without } \\
\text { GO group A }(85 \%)\end{array}$ & $\begin{array}{l}53 \text { patients with new } \\
\text { GO group B }(15 \%)\end{array}$ & $\begin{array}{l}\text { P value } \\
\text { (A vs } B \text { ) }\end{array}$ \\
\hline $\mathrm{Age}^{\dagger}$ & $42.9 \pm 13.2$ year & $42.4 \pm 13.1$ year & $45.7 \pm 13.1$ year & 0.087 \\
\hline Female/male gender & $82 \% / 18 \%$ & $84 \% / 16 \%$ & $75 \% / 25 \%$ & 0.169 \\
\hline Caucasian/other ethnicity & $94 \% / 6 \%$ & $93 \% / 7 \%$ & $96 \% / 4 \%$ & 0.550 \\
\hline Family history of AITD & $20 \%$ & $19 \%$ & $26 \%$ & 0.321 \\
\hline Other Al disease in patient & $6 \%$ & $6 \%$ & $4 \%$ & 0.750 \\
\hline Never/ex/current smokers & $58 \% / 13 \% / 29 \%$ & $60 \% / 14 \% / 26 \%$ & $43 \% / 8 \% / 49 \%$ & 0.002 \\
\hline Pack-years* & $15(7.5-24)$ & $15(7.3-22.9)$ & $20(15.5-32.5)$ & 0.051 \\
\hline Cigarettes/day ${ }^{\dagger}$ & $12.7 \pm 7.0$ & $11.5 \pm 5.9$ & $16.0 \pm 8.6$ & 0.022 \\
\hline Duration of symptoms months ${ }^{\S, *}$ & $3(1.5-5.25)$ & $3(1-5)$ & $4(2-6.25)$ & 0.007 \\
\hline Antithyroid drugs: titration/block-and-replace & $80 \% / 20 \%$ & $80 \% / 20 \%$ & $88 \% / 12 \%$ & 0.179 \\
\hline $\mathrm{TSH}(\mathrm{U} / \mathrm{L}) *$ & $0.01(0.00-0.02)$ & $0.01(0.00-0.02)$ & $0.01(0.00-0.02)$ & 0.463 \\
\hline $\mathrm{FT} 4(\mathrm{pmol} / \mathrm{L}) *$ & $36.6(27.2-51.5)$ & $36.4(27.1-50.2)$ & $39.4(29.4-59.4)$ & 0.102 \\
\hline FT3 (pmol/L)* & $13.6(8.2-22.1)$ & $13.6(8.1-22.0)$ & $13.3(8.6-23.1)$ & 0.689 \\
\hline $\mathrm{TPO}(\mathrm{kU} / \mathrm{L}) *$ & $200(46-1000)$ & $206(47-1060)$ & $164(17-600)$ & 0.141 \\
\hline TBII $(\mathrm{U} / \mathrm{L}) *$ & $6.8(3.8-13.4)$ & $6.4(3.7-12.8)$ & $11.0(5.1-21.5)$ & 0.006 \\
\hline CAS score $(0 / 1 / 2)$ & $89 \% / 10 \% /<1 \%$ & $92 \% / 7 \% /<1 \%$ & $73 \% / 25 \% / 2 \%$ & $<0.001$ \\
\hline Vancouver Orbitopathy Rule negative/positive & $98 \% / 2 \%$ & $99 \% / 1 \%$ & $94 \% / 6 \%$ & 0.075 \\
\hline Lid aperture $(\mathrm{mm})^{\dagger}$ & $9.5 \pm 1.7$ & $9.5 \pm 1.8$ & $9.6 \pm 1.7$ & 0.806 \\
\hline Proptosis $(\mathrm{mm})^{\dagger}$ & $16.9 \pm 2.2$ & $16.8 \pm 2.2$ & $17.3 \pm 2.2$ & 0.028 \\
\hline
\end{tabular}

${ }^{\S}$ Hyperthyroid symptoms until start of antithyroid drugs; ${ }^{\dagger}$ mean \pm s.D.; ${ }^{*}$ median values with interquartile range. $\mathrm{Al}$, autoimmune; AITD, autoimmune thyroid disease.

developed GO at 18 months. GO developed in 53 (15\%) of the 348 patients, which was mild in 46 patients $(13 \%)$ and moderate to severe in 7 patients (2\%). Their characteristics at baseline and at time of GO diagnosis are shown in Supplementary Table (see section on supplementary data given at the end of this article). GO developed in 26 patients at 6 months (7.4\%), in 18 patients at 12 months (5.1\%), and in 9 patients at 18 months $(2.5 \%)$ follow-up respectively. There was a direct relationship between the number of patients who developed GO and the number of patients recruited by each individual center (Fig. 1). Mixed model analysis also confirmed homogeneity between centers with respect to patient populations. Baseline characteristics of the 295 patients who did not develop GO and the 53 patients who developed GO are given in Table 1. Patients who developed GO tended to be older, had longer duration of hyperthyroid symptoms, were more often current smokers, consumed more cigarettes per day and had a higher number of pack-years. Serum TBII were also higher in patients who developed GO, but otherwise the biochemical severity of hyperthyroidism and choice for titration or block-and-replace regimen were similar in both groups. GO was further associated with a positive Vancouver Orbitopathy Rule and higher CAS scores at baseline. CAS $>0$ at baseline was observed in 36 patients: CAS of 1 was present in 34 patients and CAS of 2 in 2 patients. The distribution of the various
CAS items was as follows: mild eyelid swelling 10, plica/ caruncle swelling 0 , chemosis 0 , spontaneous pain 4 , pain on eye movement 3 , mild redness of conjunctiva 7 , redness of eyelids 14 . One patient with CAS 2 had spontaneous pain and pain on movement, while the

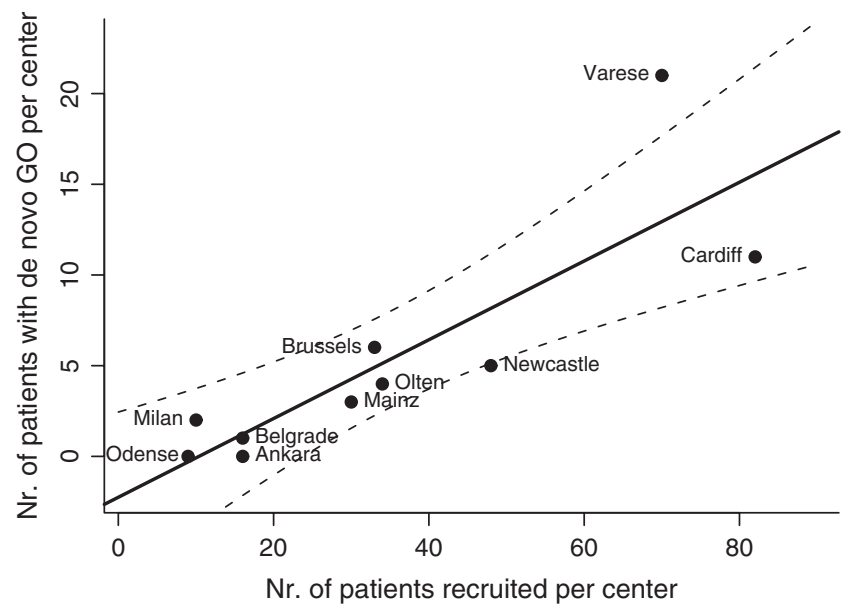

\section{Figure 1}

Direct relationship between number of patients recruited per center and number of patients developing Graves' orbitopathy (GO) per center $(r=0.85, P=0.002$ ) (AITD, autoimmune thyroid disease; Al, autoimmune; $\S$, hyperthyroid symptoms until start of antithyroid drugs; †, mean \pm s.D.; *, median values with interquartile range). 
other with CAS 2 had mild eyelid swelling and mild redness of conjunctiva. Patients with scores 1 or 2 were collapsed in a single category (CAS $>0$ ). GO developed in $12.5 \%$ of 312 patients with baseline CAS of 0 , and in $39 \%$ of 36 patients with baseline CAS $\geq 1(P<0.001)$. Development of GO was thus associated with older age, current smoking, longer duration of hyperthyroid symptoms, higher TBII, higher CAS and a positive Vancouver Orbitopathy Rule.

Using mixed-effect logistic regression analysis, four variables were identified, which were independent of each other and predicted development or progression to obvious GO: CAS $>0$, current smoking, TBII and duration of hyperthyroid symptoms; their odds ratios are listed in Table 2. In the multivariate analysis, age and the Vancouver Orbitopathy Rule were no longer independent determinants of GO. For each of the ten centers, probability of developing $\mathrm{GO}$ for the four variables is presented in Fig. 2.

A predictive score (Prediction of Graves' Orbitopathy (PREDIGO)) was constructed based on the four independent variables. Points were assigned to the presence or absence of each variable, and the sum of the points provides the numerical predictive score that ranges from 0 to 15 (Table 3). Scores $>6$ have some predictive value for developing $\mathrm{GO}$, whereas scores of $\leq 6$ are not predictive of developing GO. Sensitivity of the predictive score is 0.56 (95\% CI: $0.42-0.70)$, specificity 0.75 $(0.70-0.79)$, positive predictive value $0.28(0.20-0.37)$ and negative predictive value $0.91(0.87-0.94)$. The area under the ROC curve of the predictive score is $0.71(0.63-0.789)$ (Fig. 3). The proportion of patients who developed GO

Table 2 Baseline variables predicting development of GO in patients with Graves' hyperthyroidism treated for 18 months with antithyroid drugs.

\begin{tabular}{l}
$\begin{array}{l}\text { Baseline variable } \\
\text { predicting Go }\end{array}$ \\
\hline CAS 0 \\
CAS $\geq 1$ \\
TBII $<2(\mathrm{U} / \mathrm{L})$ \\
TBII $2-10(\mathrm{U} / \mathrm{L})$ \\
TBII $>10(\mathrm{U} / \mathrm{L})$ \\
Duration hyperthyroid \\
symptoms \\
$<1$ month \\
$1-4$ months \\
$>4$ months \\
Current smoker no \\
Current smoker yes \\
\hline
\end{tabular}

\begin{tabular}{ccr}
$\begin{array}{c}\text { Univariate odds ratio } \\
(95 \% \mathrm{Cl})\end{array}$ & & \multicolumn{1}{c}{$\boldsymbol{P}$ value } \\
\cline { 1 - 1 } $\begin{array}{c}1.00 \\
4.45(2.11-9.42)\end{array}$ & & $<0.01$ \\
1.00 & & \\
$1.82(0.93-3.54)$ & & 0.08 \\
$3.75(1.57-9.00)$ & & $<0.01$ \\
& & \\
& & \\
1.00 & & 0.16 \\
$1.62(0.82-3.19)$ & & $<0.01$ \\
$3.35(1.47-7.64)$ & & $<0.01$ \\
1.00 & \\
$2.77(1.53-5.05)$ & \\
\hline
\end{tabular}

among all patients with a particular predictive score is depicted in Fig. 4.

\section{Discussion}

\section{Rate of GO development after the onset of Graves' hyperthyroidism}

In this multicenter study, $15 \%$ of patients with newly diagnosed GH but without overt GO, developed GO
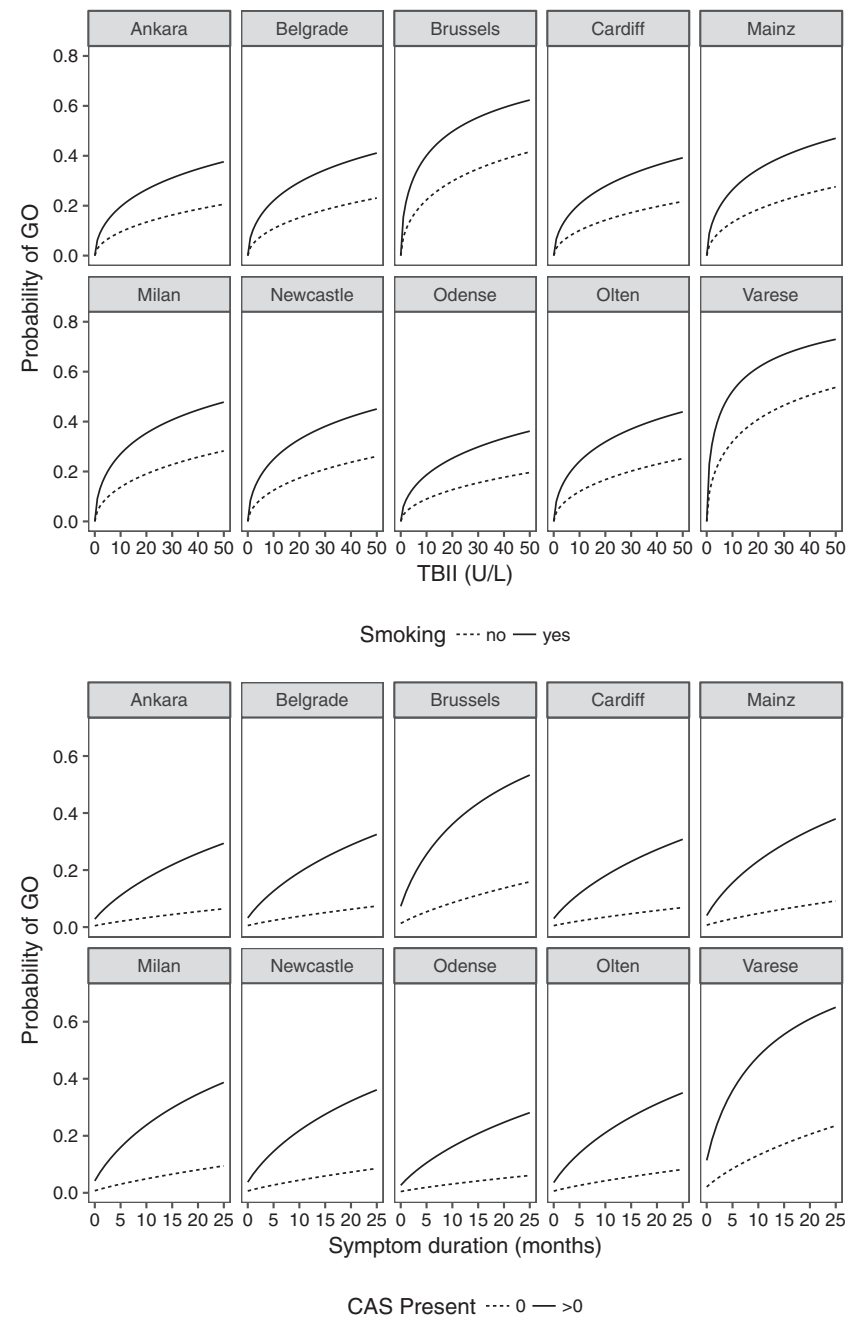

Figure 2

Probability of developing Graves' orbitopathy in patients with Graves' hyperthyroidism during 18-month treatment with antithyroid drugs per participating center, as a function of TBII and current smoking (top panels) and as a function of duration of hyperthyroid symptoms and clinical activity score (bottom panels). 
Table 3 Predictive score for development of GO (PREDIGO) in patients with Graves' hyperthyroidism treated with antithyroid drugs for 18 months.

\begin{tabular}{lccc}
\hline Baseline variable & Cut-off & Score \\
\cline { 1 - 2 } CAS & 0 & 0 \\
TBII & $<1$ & 5 \\
& $2-10(\mathrm{U} / \mathrm{L})$ & \\
Duration of hyperthyroid & $>10(\mathrm{U} / \mathrm{L})$ & \\
symptoms & $<1$ months & 0 \\
& $1-4$ months & 1 \\
Current smoking & $>4$ months & 3 \\
& No & 0 \\
& Yes & 2 \\
\hline
\end{tabular}

Maximum score 15.

during a follow-up of 18 months. The orbitopathy was mild in 13\%, moderate to severe in $2 \%$ and occurred predominantly at 6-12 months after starting antithyroid drugs. Differences between centers did not seem to play a role for the frequency of GO, as the number of patients who developed GO was directly related to the number of patients recruited from that center (Fig. 1).

The data are remarkably similar to those of a recent single-center Italian study from Varese published in 2013 (3). In that study, 194 patients with GH but without overt GO were followed for 18 months during/after treatment with antithyroid drugs. GO developed in 12.9\% (mild GO in $10.3 \%$, moderate-to-severe GO in $2.6 \%$ ), and most appeared 6-12 months after the start of treatment. At the time of diagnosis of hyperthyroidism, GO was absent in $74 \%$ and present in $26 \%$. New GO following the diagnosis of GH occurred in $9.5 \%(0.129 \times 74 \%)$, rendering a total GO frequency of $35.5 \%(26 \%+9.5 \%)$ in a population of $\mathrm{GH}$. GO was diagnosed at the same time as $\mathrm{GH}$ in $73 \%$ (26 out of 35.5), whereas GO onset followed that of hyperthyroidism in 27\% (9.5 out of 35.5 ). These figures are at variance with reports in the older literature, stating that GO develops after, simultaneously with or before $\mathrm{GH}$ in about 47,38 and $15 \%$ respectively $(20,21,22)$. The rates of GO cases developing after diagnosis of $\mathrm{GH}$ are apparently much higher in the past (47\%) than at present $(27 \%)$. Earlier diagnosis and treatment of hyperthyroidism, identification of the risks conferred by 131I therapy and post-radioiodine hypothyroidism and focus on the detrimental effects of smoking have likely contributed to this secular decline. The lower prevalence and incidence of late-onset GO seems to be the result of effective primary and secondary prevention of GO (23).

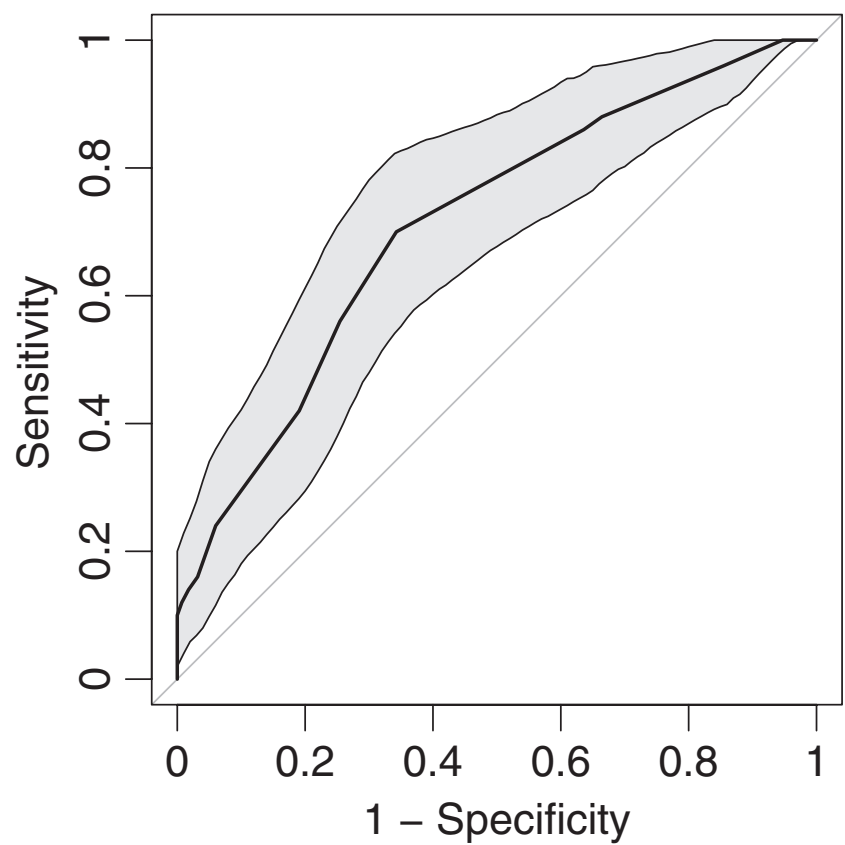

Figure 3

Receiver-operator curve with confidence intervals of the PREDIGO score for predicting development of Graves' orbitopathy in patients with Graves' hyperthyroidism treated for 18 months with antithyroid drugs.

Nowadays it is rare for a patient with GH who presents without GO to contract moderate-to-severe GO later in the course of the disease. Indeed, the clinical phenotype of Graves' disease is presently milder than that in the past $(24,25)$.

\section{Determinants of GO development}

We identified four independent determinants as predictors of GO development in patients with newly diagnosed GH: CAS, TBII, current smoking and longer duration of hyperthyroid symptoms. Associations between these items and GO are known from previous studies $(8,9,10,11$, 12). However, this is the first time the association of these items with development of GO has been demonstrated in a prospective manner in hyperthyroid patients who had no overt GO at study entrance. It is not immediately clear why a longer duration of hyperthyroid symptoms is a predictor of GO. In a previous study among 251 consecutive patients with newly diagnosed $\mathrm{GH}$, duration of symptoms $>4$ months was associated with younger age, higher TPOAb (thyroid peroxidase antibodies) levels, larger goiter size, but not with higher TBII (TSH-binding 


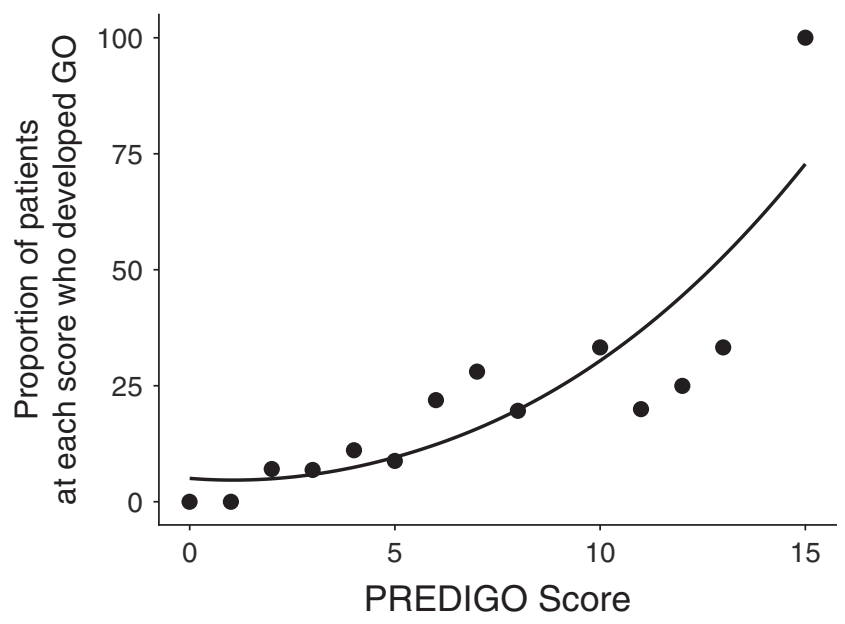

\section{Figure 4}

Predictive score for development of Graves' orbitopathy in patients with Graves' hyperthyroidism treated with antithyroid drugs for 18 months, called the PREDIGO (PREDIctor of Graves' Orbitopathy). Scores $\leq 6$ are predictive of not developing $\mathrm{GO}$, whereas scores $>6$ have some predictive value for developing GO.

inhibitor immunoglobulins) or higher prevalence of GO (although the prevalence of GO was 19\% at symptoms duration $\leq 4$ months and increased to $28 \%$ at a duration $\geq 12$ months, the trend was not significant) (26). One may hypothesize that longer duration of symptoms means longer exposure to TBII, which is associated with larger goiter size and by analogy may also lead to larger size of extraocular muscles and orbital fat.

Biochemically more severe $\mathrm{GH}$ has been associated with a higher risk of GO in a Swedish prospective randomized clinical trial (5). The slightly higher baseline serum FT4 in patients who developed GO in our study, although not statistically significant, points in the same direction.

Assessment of the extent of swelling and redness of the eyes remains subjective, and minor degrees may remain unnoticed and/or not necessarily indicate GO. We tried to increase objectivity in deciding whether eyelid swelling or conjunctival redness was absent or mild (allowing inclusion), or moderate to severe (patient excluded) with the help of a color atlas of eye changes in GO (15). Retrobulbar pain, mild eyelid swelling and/ or mild conjunctival redness count in the CAS, which proved to be one of the main independent predictors of GO development. One may argue that these minor degrees of swelling, redness and pain already indicated the presence of GO at baseline. Even so, the PREDIGO score retains its value in predicting progression of very mild (almost subclinical) GO to more overt GO. It is interesting that the Vancouver Orbitopathy Rule (asking among others also for redness in the eyes and redness or fullness of eyelids) had some predictive value in the univariate analysis, providing confirmation in an independent cohort of the association between a positive Vancouver Orbitopathy Rule and GO development (14). Its question 'Do your eyes seem to be wide open?' may reflect the slightly higher baseline values of lid aperture and proptosis in our patients who developed GO.

Other putative determinants of developing GO in our study could have been differences in the rate of control of hyperthyroidism and the extent to which euthyroidism was restored. Thyroid function tests were, however, not different between groups at 6,12 and 18 months during treatment with antithyroid drugs (data not shown). There were no differences with respect to development of GO between patients who were treated with antithyroid drugs according the block-and-replace regimen or the titration regimen.

\section{Quantitative predictive score for GO development}

Based on the four independent determinants, we were able to construct a quantitative predictive score for the development of GO, called PREDIGO. The score ranges from 0 to a maximum of 15 . Low scores are associated with a low probability of developing GO, and vice versa. PREDIGO had a high negative predictive value of 0.91 but a rather low positive predictive value of 0.28 . In other words, it seems better at identifying those patients who escape GO than those who will develop GO. Improvement of the positive predictive value may have to await the development of more sensitive biomarkers for GO, which will likely depend on better insight into the pathogenesis of the disease. It remains to be demonstrated under which circumstances the score will be most useful in the management of Graves' disease patients. One may consider studies to evaluate whether a high predictive score would alter management, e.g. in refraining from 131I therapy or starting with selenium. In case of a high predictive score, this could be used as a disease-specific argument to convince the patient to stop smoking.

\section{Strengths and limitations}

Strengths of our study relate to its prospective nature, its large sample size and the structured investigation according to a detailed protocol. Eye changes were 
recorded predominantly by endocrinologists and not by ophthalmologists. Slit lamp investigations, which are performed exclusively by ophthalmologists, are thus not assessed in the present study. We view this as an advantage rather than a disadvantage. For newly diagnosed patients with GH without overt GO are nearly always managed by internists/endocrinologists. Thereby applicability of the results of this study for routine endocrine practice may be inferred.

\section{Supplementary data}

This is linked to the online version of the paper at https://doi.org/10.1530/ EJE-18-0039.

\section{Declaration of interest}

The authors declare that there is no conflict of interest that could be perceived as prejudicing the impartiality of this study.

\section{Funding}

This research did not receive any specific grant from any funding agency in the public, commercial or not-for-profit sector.

\section{References}

1 Smith TJ \& Hegedüs L. Graves' disease. New England Journal of Medicine 2016375 1552-1565. (https://doi.org/10.1056/ NEJMra1510030)

2 Wiersinga WM. Quality of life in Graves' ophthalmopathy. Best Practice and Research Clinical Endocrinology and Metabolism 201226 359-370. (https://doi.org/10.1016/j.beem.2011.11.001)

3 Tanda ML, Piantanida E, Liparulo L, Veronesi G, Lai A, Sassi L, Pariani N, Gallo D, Azzolini C, Ferrario M et al. Prevalence and natural history of Graves' orbitopathy in a large series of patients with newly diagnosed Graves' hyperthyroidism seen at a single center. Journal of Clinical Endocrinology and Metabolism 201398 1443-1449. (https://doi.org/10.1210/jc.2012-3873)

4 Bartalena L, Marcocci C, Bogazzi F, Manetti L, Tanda ML, Dell'Unto E, Bruno-Bossio G, Nardi M, Bartolomei MP, Lepri A et al. Relation between therapy for hyperthyroidism and the course of Graves' ophthalmopathy. New England Journal of Medicine 1998338 73-78. (https://doi.org/10.1056/NEJM199801083380201)

5 Tallstedt L, Lundell G, Tørring O, Wallin G, Ljunggren JG, Blomgren $\mathrm{H} \&$ Taube A. Occurrence of ophthalmopathy after treatment for Graves' hyperthyroidism. The Thyroid Study Group. New England Journal of Medicine 1992326 1733-1738. (https://doi. org/10.1056/NEJM199206253262603)

6 Acharya SH, Avenell A, Philip S, Burr J, Bevan JS \& Abraham P. Radioiodine therapy (RAI) for Graves' disease (GD) and the effect on ophthalmopathy: a systematic review. Clinical Endocrinology 200869 943-950. (https://doi.org/10.1111/j.1365-2265.2008.03279.x)

7 Marcocci C, Kahaly GJ, Krassas GE, Bartalena L, Prummel M, Stahl M, Altea MA, Nardi M, Pitz S, Boboridis K et al. Selenium and the course of mild Graves' orbitopathy. New England Journal of Medicine 2011 364 1920-1931. (https://doi.org/10.1056/NEJMoa1012985)
8 Wiersinga WM. Smoking and thyroid. Clinical Endocrinology 201379 145-151. (https://doi.org/10.1111/cen.12222)

9 Eckstein AK, Plicht M, Lax H, Neuhäuser M, Mann K, Lederbogen S, Heckmann C, Esser J \& Morgenthaler NG. Thyrotropin receptor autoantibodies are independent risk factors for Graves' ophthalmopathy and help to predict severity and outcome of the disease. Journal of Clinical Endocrinology and Metabolism 200691 3464-3470. (https://doi.org/10.1210/jc.2005-2813)

10 Lytton SD, Ponto KA, Kanitz M, Matheis N, Kohn LD \& Kahaly GJ. A novel thyroid stimulating immunoglobulin bioassay is a functional indicator of activity and severity of Graves' orbitopathy. Journal of Clinical Endocrinology and Metabolism 201095 2123-2131. (https:// doi.org/10.1210/jc.2009-2470)

11 Perros P, Crombie AL, Matthews JNS \& Kendall-Taylor P. Age and gender influence the severity of thyroid-associated ophthalmopathy: a study of 101 patients attending a combined thyroid-eye clinic. Clinical Endocrinology 199338 367-372. (https://doi. org/10.1111/j.1365-2265.1993.tb00516.x)

12 Kendler DL. The initial clinical characteristics of Graves' orbitopathy vary with age and sex. Archives of Ophthalmology 1993111197. (https://doi.org/10.1001/archopht.1993.01090020051022)

13 Mourits MP, Prummel MF, Wiersinga WM \& Koornneef L. Clinical activity score as a guide in the management of patients with Graves' ophthalmopathy. Clinical Endocrinology 199747 9-14. (https://doi. org/10.1046/j.1365-2265.1997.2331047.x)

14 Mohaseb K, Linder M, Rootman J, Wilkins GE, Schechter MT, Dolman PJ \& Singer J. Development and validation of a patient symptom questionnaire to facilitate early diagnosis of thyroidassociated orbitopathy in Graves' disease. Orbit 200827 419-425. (https://doi.org/10.1080/01676830802414566)

15 Dickinson AJ \& Perros P. Controversies in the clinical evaluation of active thyroid-associated orbitopathy: use of a detailed protocol with comparative photographs for objective assessment. Clinical Endocrinology 200155 283-303. (https://doi.org/10.1046/j.13652265.2001.01349.x)

16 R Core Team. R: A Language and Environment for Statistical Computing. Vienna, Austria: R Foundation for Statistical Computing, 2017.

17 Bates D, Mächler M, Bolker B \& Walker S. Fitting linear mixed-effects models using lme4. Journal of Statistical Software 201567 1-48. (https://doi.org/10.18637/jss.v067.i01)

18 Sullivan LM, Massaro JM \& D'Agostino RB. Presentation of multivariate data for clinical use: the Framingham Study risk score functions. Statistics in Medicine 200423 1631-1660. (https://doi. org/10.1002/sim.1742)

19 López-Ratón M, Rodríguez-Álvarez MX, Suárez CC \& Sampedro FG. OptimalCutpoints: an R package for selecting optimal cutpoints in diagnostic tests. Journal of Statistical Software 201461 1-36. (https:// doi.org/10.18637/jss.v061.i08)

20 Wiersinga WM, Smit T, van der Gaag R \& Koornneef L. Temporal relationship between onset of Graves' ophthalmopathy and onset of thyroidal Graves' disease. Journal of Endocrinological Investigation 1988 11 615-619. (https://doi.org/10.1007/BF03350193)

21 Marcocci C, Bartalena L, Bogazzi F, Panicucci M \& Pinchera A. Studies on the occurrence of ophthalmopathy in Graves' disease. Acta Endocrinologica 1989120 473-478. (https://doi.org/10.1530/ acta.0.1200473)

22 Burch HB \& Wartofsky L. Graves' ophthalmopathy: current concepts regarding pathogenesis and management. Endocrine Reviews 199314 747-793. (https://doi.org/10.1210/edrv-14-6-747)

23 Bartalena L. Prevention of Graves' ophthalmopathy. Best Practice and Research Clinical Endocrinology and Metabolism 201226 371-379. (https://doi.org/10.1016/j.beem.2011.09.004)

24 Bartalena L, Masiello E, Magri F, Veronesi G, Bianconi E, Zerbini F, Gaiti M, Spreafico E, Gallo D, Premoli P et al. The phenotype of 
newly diagnosed Graves' disease in Italy in recent years is milder than in the past: results of a large observational longitudinal study. Journal of Endocrinological Investigation 201639 1445-1451. (https:// doi.org/10.1007/s40618-016-0516-7)

25 Perros P, Žarković M, Azzolini C, Ayvaz G, Baldeschi L, Bartalena L, Boschi A, Bournaud C, Brix TH, Covelli D et al. PREGO (presentation of Graves' orbitopathy) study: changes in referral patterns to
European Group on Graves' Orbitopathy (EUGOGO) centres over the period from 2000 to 2012. British Journal of Ophthalmology 201599 1531-1535. (https://doi.org/10.1136/bjophthalmol-2015-306733)

26 Vos XG, Smit N, Endert E, Tijssen JGP \& Wiersinga WM. Variation in phenotypic appearance of Graves' disease: effect of genetic anticipation and duration of complaints. European Journal of Endocrinology 2009161 113-118. (https://doi.org/10.1530/eje-08-0991)

Received 16 January 2018

Revised version received 21 March 2018

Accepted 11 April 2018 